International Mathematical Forum, 2, 2007, no. 58, 2859 - 2867

\title{
Harmonic Univalent Functions Defined by Ruscheweyh Derivatives
}

\author{
Abdul Rahman S. Juma \\ Department of Mathematics \\ University of Pune, Pune - 411007, India \\ absa662004@yahoo.com \\ S. R. Kulkarni \\ Department of Mathematics \\ Fergusson College, Pune - 411004, India \\ kulkarni_ferg@yahoo.com
}

\begin{abstract}
By applying Ruscheweyh derivative on the class $A S_{H}(\lambda, \alpha, k, \gamma)$ of harmonic univalent functions in the unit disk $\mathcal{U}$, we obtain several interesting properties such as sharp coefficient relations, distortion bounds, extreme points, Hadamard product, and other results.
\end{abstract}

\section{Mathematics Subject Classification: 30C45}

Keywords: Harmonic function, Univalent function, Ruscheweyh derivative, Hadamard product, Distortion bounds

\section{Introduction}

Let $\mathcal{H}$ be a class of all harmonic functions $f=h+\bar{g}$ that are univalent and sense preserving in the open $\operatorname{disk} \mathcal{U}=\{z:|z|<1\}$ where

$$
h(z)=z+\sum_{n=2}^{\infty} a_{n} z^{n} \quad \text { and } \quad g(z)=\sum_{n=1}^{\infty} b_{n} z^{n} ; \quad\left|b_{1}\right|<1
$$

normalized by $f(0)=f_{z}(0)-1=0$ with $f_{z}(0)$ denotes partial derivative of $f(z)$ at $z=0$ and we call $h$ and $g$ analytic part and co-analytic part of $f$ respectively. 
Now, we introduce a new class $A S_{H}(\lambda, \alpha, k, \gamma)$ of functions $f \in \mathcal{H}$ satisfying $\operatorname{Re}\left\{\left(1+k e^{i \gamma}\right) \frac{z^{2}\left(D^{\lambda} h(z)\right)^{\prime \prime}+z\left(D^{\lambda} h(z)\right)^{\prime}+\overline{z^{2}\left(D^{\lambda} g(z)\right)^{\prime \prime}+z\left(D^{\lambda} g(z)\right)^{\prime}}}{z\left(D^{\lambda} h(z)\right)^{\prime}-\overline{z\left(D^{\lambda} g(z)\right)^{\prime}}}+1\right\} \geq \alpha$

where $z=r e^{i \theta}, \gamma, \alpha$, and $\theta$ are real such that $0 \leq \gamma<1,0 \leq \alpha<1$,

$0 \leq k<1,0 \leq r<1$ and $D^{\lambda} f(z)$ is the Ruscheweyh derivative of $f$ and is given by $D^{\lambda} f(z)=\sum_{n=1}^{\infty} B_{n}(\lambda) c_{n} z^{n}, \lambda>-1$,

$$
B_{n}(\lambda)=\frac{(\lambda+1)(\lambda+2) \cdots(\lambda+n-1)}{(n-1) !}
$$

also

$$
D^{\lambda} f(z)=D^{\lambda} h(z)+\overline{D^{\lambda} g(z)} \quad[4]
$$

Further, let $\overline{\mathcal{H}}$ be the subfamily of $\mathcal{H}$ consisting of harmonic functions $f=h+\bar{g}$ where

$$
h(z)=z-\sum_{n=2}^{\infty} a_{n} z^{n} \text { and } g(z)=-\sum_{n=1}^{\infty} b_{n} z^{n}, \quad a_{n} \geq 0, \quad b_{n} \geq 0 .
$$

Let $\overline{A S_{H}}(\lambda, \alpha, k, \gamma)$ be the subclass of functions $f \in \overline{\mathcal{H}}$ and (2) holds true.

Special cases were studied by Kanas and Wisniowska [4], and Kanas and Srivastava [3].

\section{Main Results}

We need the following theorem due to J. M. Jahangiri [2].

Theorem 1 : Let, $f=h+\bar{g}$ where

$$
h(z)=z-\sum_{n=2}^{\infty} a_{n} z^{n} \quad \text { and } \quad g(z)=-\sum_{n=1}^{\infty} b_{n} z^{n} .
$$

Furthermore let; $\sum_{n=1}^{\infty}\left(\frac{n(n-\alpha)}{1-\alpha}\left|a_{n}\right|+\frac{n(n+\alpha)}{1-\alpha}\left|b_{n}\right|\right) \leq 2$ where $a_{1}=1$ and $0 \leq \alpha<$ 1. Then $f$ is harmonic univalent in $\mathcal{U}$ and $f \in \overline{A S_{H}}(\alpha) . \overline{A S_{H}}(\alpha)$ is the subclass of $A S_{H}(\alpha)$ consisting of harmonic convex functions of order $\alpha$.

Theorem 2 : Let $f=h+\bar{g} \in \mathcal{H}$ with

$$
\sum_{n=1}^{\infty}\left(\frac{n(n(1+k)+(1-\alpha))}{2-\alpha+k}\left|a_{n}\right|+\frac{n(n(1+k)-(1-\alpha))}{2-\alpha+k}\left|b_{n}\right|\right) B_{n}(\lambda) \leq 2
$$


where $a_{1}=1,0 \leq \alpha<1, \lambda>-1,0 \leq k<1$. Then $f$ is harmonic univalent in $\mathcal{U}$ and $f \in A S_{H}(\lambda, \alpha, k, \gamma)$.

Proof : By Theorem 1, we obtain $f$ is harmonic, since

$$
\begin{aligned}
& \sum_{n=2}^{\infty} \frac{n(n-\alpha)}{1-\alpha}\left|a_{n}\right|+\sum_{n=1}^{\infty} \frac{n(n+\alpha)}{1-\alpha}\left|b_{n}\right| \\
& \leq \sum_{n=2}^{\infty}\left(\frac{n(n(1+k)+(1-\alpha))}{2-\alpha+k}\left|a_{n}\right|+\frac{n(n(1+k)-(1-\alpha))}{2-\alpha+k}\left|b_{n}\right|\right) B_{n}(\lambda),
\end{aligned}
$$

where $0 \leq \alpha<1,0 \leq k<1, \lambda>-1$.

According to the fact that,

$$
\text { Re } w \geq \alpha \Leftrightarrow|w+1-\alpha| \geq|1+\alpha-w|, \quad(w \in \mathbb{C}, \alpha \in \mathbb{R}),
$$

for proving $f \in A S_{H}(\lambda, \alpha, k)$, it suffices to show that (2) holds. If

$$
\begin{gathered}
A(\lambda, z)=\left(1+k e^{i \gamma}\right)\left[z^{2}\left(D^{\lambda} h(z)\right)^{\prime \prime}+z\left(D^{\lambda} h(z)\right)^{\prime}+\overline{z^{2}\left(D^{\lambda} g(z)\right)^{\prime \prime}}+\overline{z\left(D^{\lambda} g(z)\right)^{\prime}}\right] \\
+\left[z\left(D^{\lambda} h(z)\right)^{\prime}-\overline{z\left(D^{\lambda} g(z)\right)^{\prime}}\right]
\end{gathered}
$$

and

$$
B(\lambda, z)=z\left(D^{\lambda} h(z)\right)^{\prime}-\overline{z\left(D^{\lambda} g(z)\right)^{\prime}} .
$$

We want to show that

$$
|A(\lambda, z)+(1-\alpha) B(\lambda, z)|-|A(\lambda, z)-(1+\alpha) B(\lambda, z)| \geq 0 .
$$


Substituting (6) and (7) in (8) we get,

$$
\begin{aligned}
& |A(\lambda, z)+(1-\alpha) B(\lambda, z)|-|A(\lambda, z)-(1+\alpha) B(\lambda, z)| \\
& =\mid\left(1+k e^{i \gamma}\right)\left[z^{2}\left(D^{\lambda} h(z)\right)^{\prime \prime}+z\left(D^{\lambda} h(z)\right)^{\prime}+\overline{z^{2}\left(D^{\lambda} g(z)\right)^{\prime \prime}}+\overline{z\left(D^{\lambda} g(z)\right)^{\prime}}\right] \\
& +\left[z\left(D^{\lambda} h(z)\right)^{\prime}-\overline{z\left(D^{\lambda} g(z)\right)^{\prime}}\right]+(1-\alpha)\left[z\left(D^{\lambda} h(z)\right)^{\prime}-\overline{z\left(D^{\lambda} g(z)\right)^{\prime}} \mid\right. \\
& -\mid\left(1+k e^{i \gamma}\right)\left[z^{2}\left(D^{\lambda} h(z)\right)^{\prime \prime}+z\left(D^{\lambda} h(z)\right)^{\prime}+\overline{z^{2}\left(D^{\lambda} g(z)\right)^{\prime \prime}}+\overline{z\left(D^{\lambda} g(z)\right)^{\prime}}\right] \\
& +\left[z\left(D^{\lambda} h(z)\right)^{\prime}-\overline{z\left(D^{\lambda} g(z)\right)^{\prime}}\right]-(1+\alpha)\left[z\left(D^{\lambda}(h(z))^{\prime}-\overline{z\left(D^{\lambda} g(z)\right)^{\prime}}\right] \mid\right. \\
& =\mid\left(1+k e^{i \gamma}\right)\left[\sum_{n=2}^{\infty} n(n-1) a_{n} B_{n}(\lambda) z^{n}+z+\sum_{n=2}^{\infty} n a_{n} B_{n}(\lambda) z^{n}\right. \\
& \left.+\sum_{n=1}^{\infty} n(n-1) \bar{b}_{n} B_{n}(\lambda) \bar{z}^{n}+\sum_{n=1}^{\infty} n \bar{b}_{n} B_{n}(\lambda) \bar{z}^{n}\right]+\left[z+\sum_{n=2}^{\infty} n a_{n} B_{n}(\lambda) z^{n}-\sum_{n=1}^{\infty} n \bar{b}_{n}(\lambda) \bar{z}^{n}\right] \\
& +(1-\alpha)\left[z+\sum_{n=2}^{\infty} n a_{n} B_{n}(\lambda) z^{n}-\sum_{n=1}^{\infty} n \bar{b}_{n} B_{n}(\lambda) \bar{z}^{n}\right] \mid \\
& -\mid\left(1+k e^{i \gamma}\right)\left[\sum_{n=2}^{\infty} n(n-1) a_{n} B_{n}(\lambda) z^{n}+z+\sum_{n=2}^{\infty} n a_{n} B_{n}(\lambda) z^{n}\right. \\
& \left.+\sum_{n=1}^{\infty} n(n-1) \bar{b}_{n} B_{n}(\lambda) \bar{z}^{n}+\sum_{n=1}^{\infty} n \bar{b}_{n} B_{n}(\lambda) \bar{z}^{n}\right]+\left[z+\sum_{n=2}^{\infty} n a_{n} B_{n}(\lambda) z^{n}-\sum_{n=1}^{\infty} n \bar{b}_{n} B_{n}(\lambda) \bar{z}^{n}\right] \\
& -(1+\alpha)\left[z+\sum_{n=2}^{\infty} n a_{n} B_{n}(\lambda) z^{n}-\sum_{n=1}^{\infty} n \bar{b}_{n} B_{n}(\lambda) \bar{z}^{n}\right] \mid \\
& =\mid\left(1+k e^{i \gamma}+2-\alpha\right) z+\sum_{n=2}^{\infty} n\left(n+n k e^{i \gamma}+2-\alpha\right) a_{n} B_{n}(\lambda) z^{n} \\
& +\sum_{n=1}^{\infty} n\left(n+n k e^{i \gamma}-2+\alpha\right) \bar{b}_{n} B_{n}(\lambda) \bar{z}^{n} \mid \\
& -\mid\left(2 k e^{i \gamma}+1-\alpha\right) z+\sum_{n=2}^{\infty} n\left(n+n k e^{i \gamma}+k e^{i \gamma}+2-\alpha\right) a_{n} B_{n}(\lambda) z^{n} \\
& +\sum_{n=1}^{\infty} n\left(n+n k e^{i \gamma}-2+\alpha\right) \bar{b}_{n} B_{n}(\lambda) \bar{z}^{n} \mid \\
& \geq(3+k-\alpha)|z|-\sum_{n=2}^{\infty} n(n+n k+2-\alpha)\left|a_{n}\right| B_{n}(\lambda)|z|^{n} \\
& -\sum_{n=1}^{\infty} n(n+n k-2+\alpha)\left|b_{n}\right| B_{n}(\lambda)|z|^{n}-\sum_{n=2}^{\infty} n(n+n k-\alpha)\left|a_{n}\right| B_{n}(\lambda)|z|^{n} \\
& -\sum_{n=1}^{\infty} n(n+n k+\alpha)\left|b_{n}\right| B_{n}(\lambda)|z|^{n} \geq 2(2-\alpha+k)|z| \times \\
& \left(1-\sum_{n=2}^{\infty} n \frac{n(1+k)+(1-\alpha)}{2-\alpha+k}\left|a_{n}\right| B_{n}(\lambda)|z|^{n-1}-\sum_{n=1}^{\infty} n \frac{n(1+k)-(1-\alpha)}{2-\alpha+k}\left|b_{n}\right| B_{n}(\lambda)|z|^{n-1}\right) \\
& \geq 0 \text { (by }(5)) \text {. }
\end{aligned}
$$

Thus $f(z) \in A S_{H}(\lambda, \alpha, k, \gamma)$. 
For sharpness consider the harmonic functions of the form

$$
f(z)=z+\sum_{n=2}^{\infty} \frac{2-\alpha+k}{n[n(k+1)+(1-\alpha)]} T_{n} z^{n}+\sum_{n=1}^{\infty} \frac{2-\alpha+k}{n[n(k+1)-(1-\alpha)]} \bar{S}_{n} \bar{z}^{n}
$$

where $\sum_{n=2}^{\infty}\left|T_{n}\right|+\sum_{n=1}^{\infty}\left|S_{n}\right|=1$.

Theorem 3 : Let $f=h+\bar{g}$ defined by (4), then the necessary and sufficient condition for the function $f$ to be in the class $\overline{A S_{H}}(\lambda, \alpha, k, \gamma)$ is that

$$
\sum_{n=1}^{\infty}\left(\frac{n(n(k+1)+(1-\alpha))}{2-\alpha+k}\left|a_{n}\right|+\frac{n(n(1+k)-(1-\alpha))}{2-\alpha+k}\left|b_{n}\right|\right) B_{n}(\lambda) \leq 2
$$

where $a_{1}=1,0 \leq \alpha<1,0 \leq k<1, \lambda>-1,0 \leq \gamma<1$ and $B_{n}(\lambda)=$ $\frac{(\lambda+1)(\lambda+2) \cdots(\lambda+n-1)}{(n-1) !}$.

Proof : Since $\overline{A S_{H}}(\lambda, \alpha, k, \gamma) \subset A S_{H}(\lambda, \alpha, k, \gamma)$, then the "necessary" part follows from Theorem 2. For the "sufficient" part, we show that (10) does not hold good implies that $f \notin \overline{A S_{H}}(\lambda, \alpha, k, \gamma)$.

Now, a function $f \in \overline{A S_{H}}(\lambda, \alpha, k, \gamma)$ if and only if

$$
\operatorname{Re}\left\{1+\left(1+k e^{i \gamma}\right) \frac{z\left(D^{\lambda} f(z)\right)^{\prime \prime}}{\left(D^{\lambda} f(z)\right)^{\prime}}\right\} \geq \alpha .
$$

Therefore,

$$
\begin{aligned}
& \operatorname{Re}\left\{\left[(2-\alpha+k)|z|-\sum_{n=2}^{\infty} n(n(1+k)+(1-\alpha))\left|a_{n}\right| B_{n}(\lambda)|z|^{n}\right.\right. \\
& \left.-\sum_{n=1}^{\infty} n(n(1+k)+(1-\alpha))\left|b_{n}\right| B_{n}(\lambda)|\bar{z}|^{n}\right] /\left[z-\sum_{n=2}^{\infty} n\left|a_{n}\right| B_{n}(\lambda)|z|^{n}\right. \\
& \left.\left.+\sum_{n=1}^{\infty} n\left|b_{n}\right| B_{n}(\lambda)|\bar{z}|^{n}\right]\right\} \geq 0 .
\end{aligned}
$$

The last inequality must hold for all $z,|z|=r<1$.

Choosing the values of $z$ on the positive real axis where $0<|z|=r<1$ we must have,

$$
\begin{gathered}
{\left[(2-\alpha+k)-\sum_{n=2}^{\infty} n(n(1+k)+(1-\alpha))\left|a_{n}\right| B_{n}(\lambda) r^{n-1}\right.} \\
\left.-\sum_{n=1}^{\infty} n(n(1+k)-(1-\alpha))\left|b_{n}\right| B_{n}(\lambda) r^{n-1}\right] /\left[1-\sum_{n=2}^{\infty} n\left|a_{n}\right| B_{n}(\lambda) r^{n-1}\right.
\end{gathered}
$$




$$
\left.+\sum_{n=1}^{\infty} n\left|b_{n}\right| B_{n}(\lambda) r^{n-1}\right] \geq 0
$$

We note that if the condition (10) does not hold then the numerator in (11) when $r$ goes to 1 is negative. This contradiction for $f(z) \in \overline{A S_{H}}(\lambda, \alpha, k, \gamma)$ and the proof is complete.

\section{Extreme Point and Distortion Bounds}

Definition 3.1 [1] Let $X$ be a topological vector space over the field of complex numbers, and let $E$ be a subset of $X$, then the closed convex hull of $E$ denoted by $\operatorname{clco}(E)$ is the smallest convex set containing $E$.

Theorem 4:f $f$ clco $\overline{A S_{H}}(\lambda, \alpha, k, \gamma)$ if and only if,

$$
f(z)=\sum_{n=1}^{\infty}\left(T_{n} h_{n}(z)+S_{n} g_{n}(z)\right)
$$

where, $h_{1}(z)=z$,

$$
\begin{aligned}
h_{n}(z) & =z-\frac{2+k-\alpha}{n(n(1+k)+(1-\alpha)) B_{n}(\lambda)} z^{n} ; \quad n=2,3,4, \cdots \\
g_{n}(z) & =z-\frac{2+k-\alpha}{n(n(1+k)-(1-\alpha)) B_{n}(\lambda)} \bar{z}^{n} ; \quad n=1,2,3,4, \cdots \\
\sum_{n=1}^{\infty}\left(T_{n}+S_{n}\right) & =1, T_{n} \geq 0 \text { and } S_{n} \geq 0 .
\end{aligned}
$$

In particular the extreme points of $\overline{A S_{H}}(\lambda, \alpha, k, \gamma)$ are $\left\{h_{n}\right\}$ and $\left\{g_{n}\right\}$. Proof : Let $f$ be written as (12), then we have

$$
\begin{aligned}
f(z)= & \sum_{n=1}^{\infty}\left(T_{n}+S_{n}\right) z-\sum_{n=2}^{\infty} \frac{2+k-\alpha}{n(n(1+k)+(1-\alpha)) B_{n}(\lambda)} T_{n} z^{n} \\
& -\sum_{n=1}^{\infty} \frac{2+k-\alpha}{n(n(1+k)-(1-\alpha)) B_{n}(\lambda)} S_{n} \bar{z}^{n}=z-\sum_{n=2}^{\infty} a_{n} z^{n}-\sum_{n=1}^{\infty} b_{n} \bar{z}^{n} .
\end{aligned}
$$

Therefore,

$$
\begin{aligned}
& \sum_{n=2}^{\infty} \frac{n(n(1+k)+(1-\alpha)) B_{n}(\lambda)}{2+k-\alpha}\left|a_{n}\right|+\sum_{n=1}^{\infty} \frac{n(n(1+k)-(1-\alpha)) B_{n}(\lambda)}{2+k-\alpha}\left|b_{n}\right| \\
& =\sum_{n=2}^{\infty} T_{n}+\sum_{n=1}^{\infty} S_{n}=1-T_{n} \leq 1,
\end{aligned}
$$

and so $f \in \operatorname{clco} \overline{A S_{H}}(\lambda, \alpha, k, \gamma)$. 
Conversely, assume that $f \in \operatorname{clco} \overline{A S_{H}}(\lambda, \alpha, k, \gamma)$. Putting

$$
T_{n}=\frac{n(n(1+k)-(1-\alpha)) B_{n}(\lambda)}{2+k-\alpha}\left|a_{n}\right|, \quad n=2,3, \cdots
$$

and

$$
S_{n}=\frac{n(n(k+1)-(1-\alpha)) B_{n}(\lambda)}{2+k-\alpha}\left|b_{n}\right|, \quad n=1,2, \cdots
$$

where, $\sum_{n=1}^{\infty} T_{n}+S_{n}=1$, then we obtain by simple calculations

$$
f(z)=\sum_{n=1}^{\infty}\left(T_{n} h_{n}(z)+S_{n} g_{n}(z)\right)
$$

and the proof is complete.

Theorem 5 : Let $f \in \overline{A S_{H}}(\lambda, \alpha, k, \gamma)$, then

$$
|f(z)| \leq\left(1+\left|b_{1}\right|\right) r+\frac{1}{2 B_{2}(\lambda)}\left[\frac{2+k-\alpha}{(3-\alpha+2 k)}-\frac{k+\alpha}{(3-\alpha+2 k)}\left|b_{1}\right|\right] r^{2},|z|=r<1
$$

and

$$
|f(z)| \geq\left(1-\left|b_{1}\right|\right) r-\frac{1}{2 B_{2}(\lambda)}\left[\frac{2+k-\alpha}{(3-\alpha+2 k)}-\frac{k+\alpha}{(3-\alpha+2 k)}\left|b_{1}\right|\right] r^{2},|z|=r<1 .
$$

Proof : We have

$$
\begin{aligned}
|f(z)| \leq & \left(1+\left|b_{1}\right|\right) r+\sum_{n=2}^{\infty}\left(\left|a_{n}\right|+\left|b_{n}\right|\right) r^{n} \\
\leq & \left(1+\left|b_{1}\right|\right) r+\sum_{n=2}^{\infty}\left(\left|a_{n}\right|+\left|b_{n}\right|\right) r^{2} \\
= & \left(1+\left|b_{1}\right|\right) r+\frac{2+k-\alpha}{2(3-\alpha+2 k) B_{2}(\lambda)} \sum_{n=2}^{\infty}\left[\frac{2(3-\alpha+2 k)}{2+k-\alpha}\left|a_{n}\right|\right. \\
& \left.+\frac{2(1+\alpha+2 k)}{2+k-\alpha}\left|b_{n}\right|\right] B_{n}(\lambda) r^{2} \\
& \leq\left(1+\left|b_{1}\right|\right) r+\frac{2+k-\alpha}{2(2-\alpha+3 k) B_{2}(\lambda)}\left(1-\frac{k+\alpha}{1+2 k-\alpha}\left|b_{1}\right|\right) r^{2} \\
= & \left(1+\left|b_{1}\right|\right) r+\frac{1}{B_{2}(\lambda)}\left(\frac{2+k-\alpha}{2(3-\alpha+2 k)}-\frac{k+\alpha}{2(3-\alpha+2 k)}\left|b_{1}\right|\right) r^{2} .
\end{aligned}
$$

The next inequality can be proved by using similar arguments. 


\section{Hadamard Product and Other Properties}

Definition 4.1 : Let

$$
f(z)=z-\sum_{n=2}^{\infty}\left|a_{n}\right| z^{n}+\sum_{n=1}^{\infty}\left|b_{n}\right| \bar{z}^{n} \text { and } g(z)=z-\sum_{n=2}^{\infty}\left|c_{n}\right| z^{n}+\sum_{n=1}^{\infty}\left|d_{n}\right| \bar{z}^{n} .
$$

We define the convolution of $f(z)$ and $g(z)$ as

$$
(f * g)(z)=f(z) * g(z)=z-\sum_{n=2}^{\infty}\left|a_{n}\right|\left|c_{n}\right| z^{n}+\sum_{n=1}^{\infty}\left|b_{n}\right|\left|d_{n}\right| \bar{z}^{n}
$$

Theorem 6 : Let $f(z) \in \overline{A S_{H}}(\lambda, \alpha, k, \gamma)$ and $g(z) \in \overline{A S_{H}}(\lambda, \beta, k, \gamma)$, then for $0 \leq \beta \leq \alpha<1$, we have

$$
(f * g)(z) \in \overline{A S_{H}}(\lambda, \alpha, k, \gamma) \subset \overline{A S_{H}}(\lambda, \beta, k, \gamma) .
$$

Proof : Since $f(z) \in \overline{A S_{H}}(\lambda, \alpha, k, \gamma)$ and $g(z) \in \overline{A S_{H}}(\lambda, \beta, k, \gamma)$, then both of them satisfy (10) and since $\left(\left|c_{n}\right| \leq 1,\left|d_{n}\right| \leq 1\right)$, we can write

$$
\begin{aligned}
& \sum_{n=1}^{\infty}\left(\frac{n(n(1+k)+(1-\alpha))}{2-\alpha+k}\left|a_{n} c_{n}\right|+\frac{n(n(1+k)-(1-\alpha))}{2-\alpha+k}\left|b_{n} d_{n}\right|\right) B_{n}(\lambda) \\
& \leq \sum_{n=1}^{\infty}\left(\frac{n(n(1+k)+(1-\alpha))}{2-\alpha+k}\left|a_{n}\right|+\frac{n(n(1+k)-(1-\alpha))}{2-\alpha+k}\left|b_{n}\right|\right) B_{n}(\lambda) .
\end{aligned}
$$

The right hand side of the last inequality is bounded above by 1 , then

$$
f * g \in \overline{A S_{H}}(\lambda, \alpha, k, \gamma) \subset \overline{A S_{H}}(\lambda, \beta, k, \gamma) .
$$

The proof of this theorem is complete.

Theorem 7 : The class $\overline{A S_{H}}(\lambda, \alpha, k, \gamma)$ is closed under convex combinations.

Proof : Suppose $f_{i} \in \overline{A S_{H}}(\lambda, \alpha, k, \gamma)(i=1,2, \cdots)$ are defined by

$$
f_{i}(z)=z-\sum_{n=2}^{\infty}\left|a_{i, n}\right| z^{n}-\sum_{n=1}^{\infty}\left|b_{i, n}\right| \bar{z}^{n}
$$

From (10), we have

$$
\sum_{n=1}^{\infty}\left(\frac{n(n(1+k)+(1-\alpha))}{2-\alpha+k}\left|a_{i, n}\right|+\frac{n(n(1+k)-(1-\alpha))}{2-\alpha+k}\left|b_{i, n}\right|\right) B_{n}(\lambda) \leq 2
$$

For $\sum_{i=1}^{\infty} S_{i}=1,0 \leq S_{i} \leq 1$, we may write the convex combination of $f_{i}$ as

$$
\sum_{i=1}^{\infty} S_{i} f_{i}(z)=z-\sum_{n=2}^{\infty}\left(\sum_{i=1}^{\infty} S_{i}\left|a_{i, n}\right|\right) z^{n}-\sum_{n=1}^{\infty}\left(\sum_{i=1}^{\infty} S_{i}\left|b_{i, n}\right|\right) \bar{z}^{n}
$$


Thus

$$
\begin{aligned}
& \sum_{n=1}^{\infty}\left[\frac{n(n(1+k)+(1-\alpha))}{2-\alpha+k}\left(\sum_{i=1}^{\infty} S_{i}\left|a_{i, n}\right|\right)+\frac{n(n(1+k)-(1-\alpha))}{2-\alpha+k}\left(\sum_{i=1}^{\infty} S_{i}\left|b_{i, n}\right|\right)\right] B_{n}(\lambda) \\
& =\sum_{i=1}^{\infty}\left\{\left[\sum_{n=1}^{\infty} \frac{n(n(1+k)+(1-\alpha))}{2-\alpha+k}\left|a_{i, n}\right|+\frac{n(n(1+k)(1-\alpha))}{2-\alpha+k}\left|b_{i, n}\right|\right] B_{n}(\lambda)\right\} S_{i} \\
& \leq 2 \sum_{i=1}^{\infty} S_{i}=2
\end{aligned}
$$

then $\sum_{i=1}^{\infty} S_{i} f_{i}(z) \in \overline{A S_{H}}(\lambda, \alpha, k \gamma)$.

\section{REFERENCES}

[1] P. L. Duren, Univalent functions, Grundlehren der Mathematischen Wissenchaften 259, Springer-Verlag, New York, Berlin, Heidelberg, Tokyo, 1983.

[2] J. M. Jahangiri, Coefficient bounds and univalence criteria for harmonic functions with negative coefficients, Ann. University Mariae Curie-Sklodowska Sect. A. 52, No. 2, (1998), 57-66.

[3] S. Kanas and H. M. Srivastava, Linear operator associated with $k$-uniformly convex functions, Integral Transform. Spec. Funct., 9(2) (2000), 121-132.

[4] S. Kanas and A. Wisniowska, Conic regions and $k$-uniform convexity, S. Comp. Appl. Math., 105 (1999), 327-336.

[5] S. M. Khairnar, Properties of harmonic convex functions in the unit disc, Ultra Science 15 (2) M, (2003), 157-164.

[6] G. Murugusundaramoorthy and K. Vijaya, On certain classes of harmonic functions involving Ruscheweyh derivatives, Bull. Cal. Math. Soc. 96(2), 99-108.

[7] S. Ruscheweyh, New criteria for univalent functions, Proc. Amer. Math. Soc. 49 (1975), 109-115.

Received: May 23, 2007 